\title{
Field Modelling of Non-Charring Flame Spread
}

\author{
MJ Lewis, PA Rubini and JB Moss \\ School of Mechanical Engineering \\ Cranfield University, Bedford, England
}

\begin{abstract}
The paper describes a methodology for the field modelling of non-charring flame spread over representative solid surfaces. Coupled descriptions of the controlling mechanisms of buoyancydriven flow, combustion, soot production, radiation heat transfer and solid pyrolysis are incorporated into a fire-specific CFD code. The approach is evaluated at sub-model level and in a room corner fire configuration through detailed comparisons between numerical prediction and experimental measurement for flame spread over PMMA reported in the literature. A range of alternative models, embracing, in particular, soot production and radiation heat transfer, are evaluated. Generally good agreement is achieved with experiment at levels of spatial resolution which are compatible with the computational demands of field model predictions in compartment fire scenarios.
\end{abstract}

KEYWORDS : Fire physics, field modelling, flame spread

\section{INTRODUCTION}

Numerical simulation, through the medium of fire consequence modelling, has established an influential position in the assessment of novel building design and the evaluation of the performance of fire safety features. Although field modelling ( computational fluid dynamics ) has made significant contributions to the analysis of smoke movement in buildings of increasingly complex layout, the underlying rates of flame spread and fire growth are typically prescribed, rather than predicted in the course of simulation. The scenarios that can then be analysed are necessarily constrained in terms of transient fire development. Whilst the levels of accessible computer resource or our knowledge of material properties may limit specific applications, the principal constraints on more detailed simulation are shortcomings in our understanding of the interaction between the critical processes - gas phase combustion, buoyancy-driven flow, radiation heat transfer and solid fuel pyrolysis, for example - and their representation in mathematically tractable form. This paper describes a general methodology in 
which the mechanisms controlling flame spread over representative solid surfaces are incorporated into a fire-specific CFD code. The approach is evaluated at sub-model level and in a room corner configuration, through detailed comparisons between numerical prediction and experimental measurement.

Flame spread over plane surfaces has attracted considerable research attention, both theoretical and experimental, over many years and these studies have been ably reviewed by FernandezPello [1]. The focus on developing an improved physical understanding of the complex processes underlying flame spread and, beyond that, on their projected inclusion in zone models of room fires in the form of correlated relationships has prompted a number of twodimensional, semi-analytical treatments. In general, the representation of solid pyrolysis is simplified to the extent that a critical pyrolysis temperature is defined at which combustible gases are released and, in the case of charring materials, a char layer is formed ahead of the pyrolysing material, shielding the virgin material from part of the external heat flux. Transient heating of the solid up to the pyrolysis temperature may be described by solutions to the onedimensional heat conduction equation [2] , relating, for example, the surface temperature, $T_{s}$, to the incident heat flux, $\dot{Q}$.

$$
T_{s}(t)-T_{0}=\left(\frac{\kappa}{\lambda^{2} \pi}\right)^{1 / 2} \int_{0}^{t} \dot{Q}(t-\tau) \frac{d \tau}{\tau^{1 / 2}}
$$

The incident heat flux must then be prescribed, however, rather in the manner of the cone calorimeter test method [3]. This introduces a measure of empiricism into such models which restricts their generality and much of the strength of the field modelling approach lies in the opportunity it provides to capture the dynamic coupling of transient gas phase behaviour and solid material properties.

As confidence in the capabilities of field models to reproduce key features of buoyant combustion flows in compartment fires has grown and the sensitivity to the treatment of combustion heat release and radiative exchange is demonstrated $[4,5,6]$, attention increasingly focusses on the fire source and the properties of combustible materials. Opstad [7] describes a CFD-based methodolgy in which the transient heating of the solid is neglected in favour of the direct incorporation of cone calorimeter data on rate of heat release versus radiative exposure. Though thereby admitting application to a wide range of materials, the implied time to ignition - surface temperature attains the pyrolysis temperature - may be substantially in error with important implications for fire growth more generally. In contrast, Yan and Holmstedt [8] present a more comprehensive treatment of two-dimensional upward flame spread over a vertical PMMA slab. A fine computational mesh is laid over the solid, on which the transient heat conduction equation, modified to include pyrolysing mass loss, is solved. The heat flux incident on the material surface then incorporates radiative feedback from the burning products of solid pyrolysis. Radiative exchange is dominated by soot formed in the hot, fuel-rich regions adjacent to the flame zone, however, and this is an important aspect of the combustion modelling which these authors do not address. Alternative approaches to soot modelling in these circumstances will be considered in this paper. 


\section{FIELD MODELLING}

The field modelling of turbulent flame spread comprises a number of distinct, but inter-related, model elements - notably, those for the buoyant flowfield, combustion heat release, soot formation, radiative exchange and thermal pyrolyis of the solid. The key features of each of these, specific to the flame spread application, will be briefly described.

\section{Flowfield Model}

The flame spread simulation described in this paper has been undertaken within the SOFIE code ( Lewis et al [6]). The modelling of buoyant turbulent flow incorporates a two-equation $(k-\varepsilon)$ turbulence model, with additional buoyancy-driven production in the equation for turbulent kinetic energy, $k$. The boundaries of the computational domain, including internal blockages, permit the conjugate solution for heat transfer in the solid. The wall functions which permit the calculation of near-wall turbulent transport of heat and momentum without unacceptable levels of mesh refinement are modified over the combustible solid to accommodate mass loss through pyrolysis. Outgassing at the surface is treated in the same manner as a transpiring wall and the approach proposed by Spalding [9] has been adopted here. In particular, the simplified closed form solutions for normalised streamwise velocity, $u_{+} \equiv u / \sqrt{\left(\tau_{s} / \rho\right)}$, and surface mass flux, $m_{+} \equiv \dot{m}_{s} / \sqrt{\left(\rho \tau_{s}\right)}$, in laminar and turbulent flow are employed, with partitioning between the two descriptions dependent upon the normalised distance from the wall, $y_{+} \equiv y \sqrt{\left(\rho \tau_{s}\right)} / \mu \leq 11.5$

\section{Combustion Model}

In these simulations, combustion heat release is assumed to be mixing controlled. The material selected for model demonstration purposes is PMMA, reflecting the wealth of experimental data available for comparison between prediction and experiment. PMMA is assumed to thermally pyrolyse simply to the monomer $\mathrm{C}_{5} \mathrm{H}_{8} \mathrm{O}_{2}$ and, at its simplest, chemical reaction then proceeds through the single step

$\mathrm{C}_{5} \mathrm{H}_{8} \mathrm{O}_{2}+6 \mathrm{O}_{2} \Rightarrow 5 \mathrm{CO}_{2}+4 \mathrm{H}_{2} \mathrm{O}$

at the rate determined by turbulent mixing from the eddy break-up expression

$$
R_{\text {fuel }}=-\rho \frac{\varepsilon}{k}\left[C_{R} m_{\text {fuel }}, C_{R} \frac{m_{o x}}{s}\right]_{\text {min }} \text {. }
$$

The products of combustion arising from this reaction are supplemented by particulate carbon in the form of soot from a separate flamelet-based mechanism.

\section{Soot Formation}

Radiative exchange between hot combustion products and the virgin combustible solid is a critical aspect of flame spread and incandescent soot particles, in turn, are the principal contributors to the radiative flux. From a modelling perspective, the simplest approach is to decouple the complex kinetic processes of soot formation and oxidation from the radiation heat transfer by prescribing the distribution of soot mass concentration. Smoke is often considered to be an inert tracer in the field modelling of building fires, which is simply transported by the buoyantly-driven flow and can therefore be linearly related to the local mixture fraction, $\zeta \quad\left(=(1+\text { AFR })^{-1}\right.$, where AFR denotes air-fuel mass ratio $)$. Such a 
representation is evidently less satisfactory in the immediate neighbourhood of the fire source where the smoke is being generated since the mixture fraction ( and hence the 'soot tracer') is greatest in the pure fuel. In the case of a pyrolysing solid material this implies that the soot concentration is also greatest immediately adjacent to the outgassing solid, although the temperature is too low there for significant soot formation $(<1400 \mathrm{~K})$.

Fully coupled models of soot production and radiative exchange have been applied to gaseous turbulent jet flames [10] and the approach is extended here to flame spread over perspex using the simplified model of soot formation for the MMA monomer proposed by Moss and Stewart [11]. Simplified expressions for the key processes of particle nucleation from the gas phase, collisional coagulation, surface mass growth from gas phase material and oxidation have been identified from detailed comparisons between numerical simulation and laminar diffusion flame experiments, burning the evaporated liquid monomer. The mechanism is incorporated in a library of flamelet state relationships - soot source term versus mixture fraction - for varying levels of radiative heat loss, $\chi_{R}$ ( $\mathrm{cf}$. fig. 1 ). The composition field is assumed to be invariant with heat loss, however, and derived from a computed adiabatic laminar flame. The most appropriate enthalpy flamelet, $h\left(\xi ; \chi_{R}\right)$ and hence soot source term, is established locally throughout the flowfield by comparing the mixture enthalpy there, $\tilde{h}(\underline{x})$, computed from a balance equation with those derived by integration over the mixture fraction pdf, $\widetilde{P}(\xi ; \underline{x})$,

$\widetilde{h}\left(\underline{x} ; \chi_{R}\right)=\int_{0}^{1} \widetilde{P}(\xi ; \underline{x}) h\left(\xi ; \chi_{R}\right) d \xi$

( cf. [12]). The soot field is characterised by two variables, the soot volume fraction $f_{l}$, and the number density $n$, which are computed from separate balance equations incorporating the flamelet-based source terms. Comparisons are reported between this latter approach and the simply prescribed soot source based on a fractional yield.

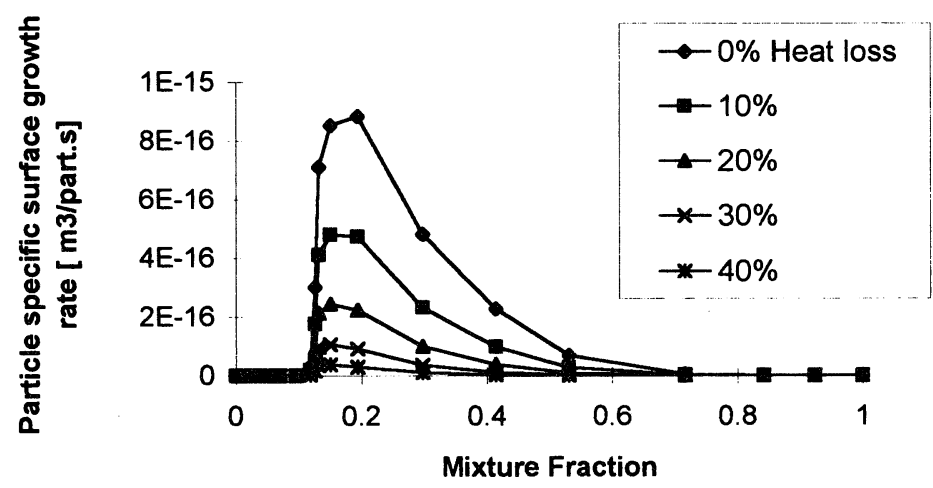

Figure 1 : Soot surface growth rates for MMA incorporating varying heat loss 


\section{Radiation Heat Transfer}

Radiative exchange is modelled using the discrete transfer (DT) radiation model [13]. DT employs a ray tracing procedure whereby the radiative transfer equation is solved along a finite number of ray paths determined by an a priori discretisation of the solid angle hemisphere normal to boundary cell faces. The change in radiative flux across an individual control volume is derived from the net balance between emission and absorption for all rays traversing that control volume. Summation of the final intensity at the end of a line-of-sight in all directions then yields the incident flux on the boundary face. For grey boundaries the radiosity is represented by a combination of the emitted intensity and the reflected component of the incident intensity. The optical properties of the combustion products $\left(\mathrm{CO}_{2}, \mathrm{H}_{2} \mathrm{O}\right.$ and soot $)$ are modelled using the weighted sum of grey gases approach, where the total emissivity is represented by

$$
\varepsilon=\sum_{j} a_{j}(T)\left\{1-\exp \left[-k_{j}\left(p_{H_{2} \mathrm{O}}+p_{C_{2}}\right)-k_{j} \rho_{s .} f_{V}\right]\right\}
$$

and where $p_{\mathrm{H}_{2} \mathrm{O}}, \quad p_{\mathrm{CO}_{2}}$ denote the partial pressures of the gaseous combustion products and $\rho_{S} f_{V}$ is the soot mass concentration. The empirical coefficients $a_{j}, k_{j}$ employed in this formulation are those reported by Truelove [14], embracing product compositions with $\mathrm{C} / \mathrm{H}$ ratios appropriate to methane $\left(\mathrm{CH}_{4}\right)$ and fuel oil $\left(\mathrm{CH}_{2}\right)$.

\section{Pyrolysis Model}

By extending the computational mesh on which the flow equations are solved into the solid at domain boundaries and internal blockages, wall heat transfer can be treated in a conjugate manner. This approach has been extended here to permit the introduction of a non-charring pyrolysis model. The time-dependent heat conduction equation, applicable in the solid, is modified to encompass energy transfer from the solid surface into the gas phase in the form of a heat of pyrolysis. In particular, in the surface layer,

$\rho c_{p} \frac{\partial T}{\partial t}=-\dot{m}_{p} H_{p}+\frac{\partial}{\partial x}\left(\lambda \frac{\partial T}{\partial x}\right)$

where the mass flux of pyrolysing solid material, $\dot{m}_{p}$, absorbs the heat of pyrolysis reaction, $H_{p}$, once the material temperature reaches a prescribed level, $T_{p}$.

Initially the net incident heat flux falling on virgin material

$$
\begin{aligned}
\dot{Q}_{\text {net }} & =\dot{Q}_{R}+\dot{Q}_{c}-\dot{Q}_{R R} \\
& \text { (radiation) (convection) }) \text { (re-radiation) }
\end{aligned}
$$

simply raises the surface temperature and heats the interior of the solid by conduction. Once the critical temperature is attained, internal conduction continues but evaporated monomer is also outgassed, acting as a fuel source and transporting its heat of formation at the pyrolysis temperature into the flowfield. Within the computational domain the gas/solid interface remains fixed in space with the cumulative mass loss used to determine local solid fuel exhaustion. 
The solid material selected for purposes of model development and evaluation is PMMA. In addition to reasonably well-established physical and chemical properties, including the soot formation mechanism based on burning the monomer as outlined earlier, there are a number of comparatively well-documented flame spread experiments in two-dimensional and corner fire configurations. In the simulations reported here the pyrolysis temperature is taken to be $600 \mathrm{~K}$, with a heat of pyrolysis of $1.6 \mathrm{MJ} \mathrm{kg}^{-1}[15]$ and with additional state and transport properties drawn from Quintiere and Rhodes [16].

\section{RESULTS AND DISCUSSION}

Two test cases are examined in detail : the two-dimensional upward flame spread experiment of Orloff et al [15] and the half-scale room corner fire experiment reported by Qian et al[17].

In the two-dimensional experiment, flame spread over a PMMA slab, $4.5 \mathrm{~cm}$ thick and $157 \mathrm{~cm}$ high, is characterised by measurements of the position of the advancing fuel vaporisation front, from which a flame spread velocity is inferred; and by measurements of the rate of mass loss from the slab mid-plane, on extinguishing the burning at discrete times, and of radiant flux normal to the surface. A number of model variants are examined in detail by Lewis [18] within the field modelling framework introduced earlier but, given the critical role of radiation heat transfer to the fuel surface, we focus here, in particular, on the sensitivity of the predictions to the sooting combustion model and to the description of radiation properties.

Computational economy plays a large part in the selection of component sub-models for field modelling applications. The weighted sum of grey gases (WSGG) approach to the determination of radiation properties offers considerable advantages over more accurate narrow band descriptions in terms of execution time - though Yan and Holmstedt [19] have recently reported a more efficient approach to narrow band computation also using precomputed look-up tables. More commonly the WSGG method is employed in the total emissivity formulation identified in eq. (4), Bressloff et al [10] demonstrate, however, that greater accuracy is achieved if the banded grey gas representation is preserved throughout the ray tracing procedure. A separate transfer equation is then solved for each grey gas.

A baseline computation has been defined for model comparison purposes on a $48 \times 56$ mesh, with 20 of the 48 transverse cells located within the solid, increasing in density towards the solid surface. The calculation employs the eddy break-up combustion model, a prescribed soot source corresponding to $2 \%$ of the pyrolysed mass flux released, the banded WSGG radiation model, with coefficients for methane, and ray tracing based on discretisation of the solid angle hemisphere into four polar and eight azimuthal divisions. The resulting 32 rays are supplemented by a single ray normal to the surface. Figure 2 compares the variation with height of the predicted flame spread velocity with the measurements of Orloff et al [15]. The irregularity in the predicted values reflects the underlying discretisation of the solid, where the progressive ignition criterion is based on surface temperature and hence on the average face temperature at each surface node. The apparent rate of flame spread is generally underpredicted although the mass release rate and incident radiative flux illustrated in fig. 3 are overpredicted by of order $20 \%$, once a steady state has been achieved and the whole of the slab is engaged. 


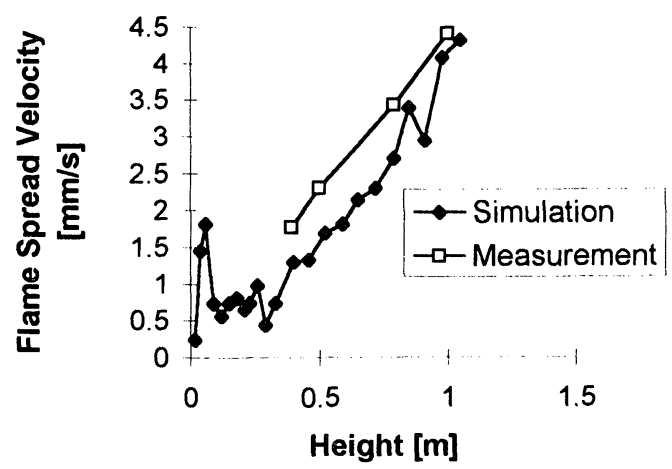

Figure 2 : Comparison between predicted flame spread velocity and measurement [ Orloff et al [15] ].
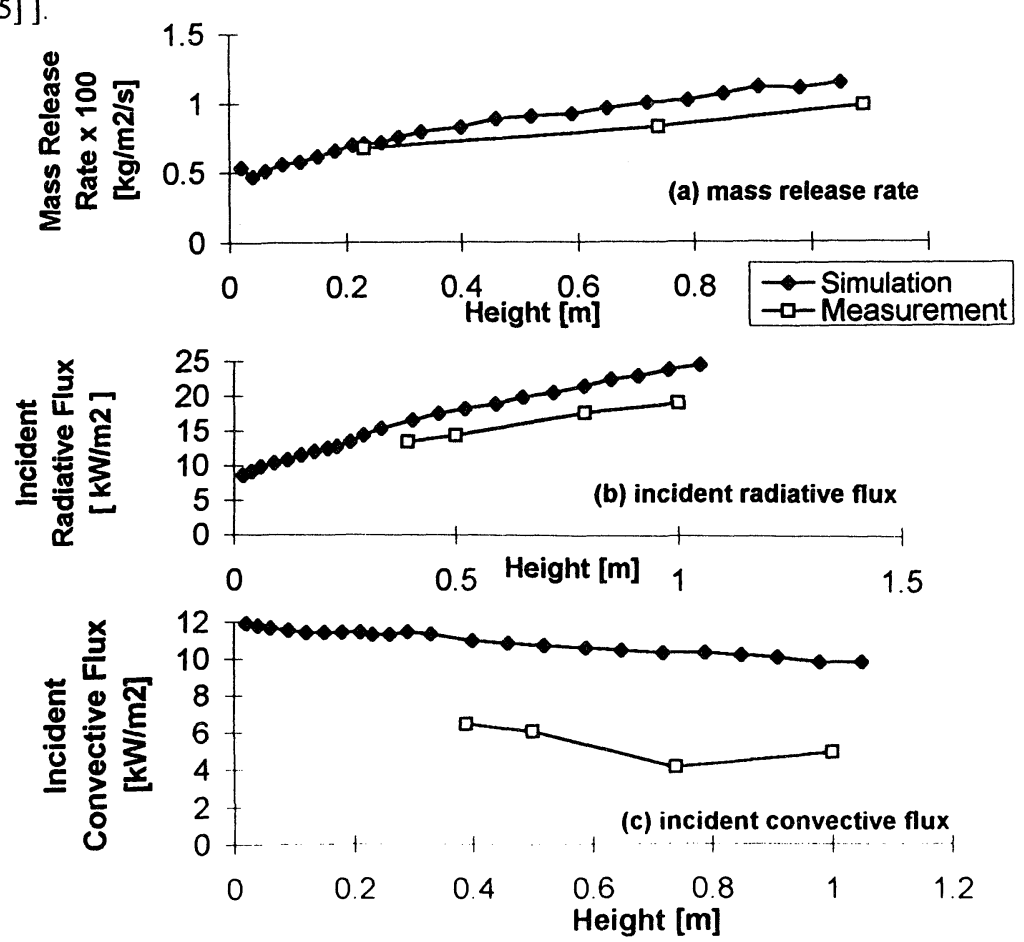

Figure 3 : Comparison between prediction and the experimental measurements of Orloff et al [15]

Orloff et [15] also tabulate estimates of the convective heat transfer to the PMMA slab, derived by difference, from direct radiation measurements and calorimetric analysis of the fuel vapour released from the surface. These values are compared with numerical prediction in fig. $3 \mathrm{c}$, revealing a substantial over-prediction. 
Whilst the derivation of experimental data by difference is prone to cumulative error, a major source of uncertainty resides in the transpiring wall function. The outgassing of the PMMA fuel surface is an intermittent and comparatively violent process which leaves the surface extensively cratered. In heat transfer applications the cooling effectiveness of transpiring walls is strongly influenced by detailed aspects of blowing momentum and discrete jet trajectories. Some re-calibration of such transpiration expressions for flame spread applications is to be expected but the existing experimental basis on which to undertake this appears to be unacceptably small. A measure of the sensitivity of the predictions to model detail is provided by the comparison of predicted incident radiative flux in fig.4. The baseline calculation is here compared with steady burning predictions which incorporate an increased number of rays in the solution for radiative exchange, in particular, introducing 16 azimuthal divisions and hence 65 rays ; adopting the absorption coefficient data appropriate to fuel oil $\left(\mathrm{CH}_{2}\right)$ in the WSGG approximation; and replacing the eddy break-up combustion model with a conserved scalar pdf - laminar flamelet formulation. Refinement of the radiation calculation brings the baseline incident flux down to the levels measured experimentally. However, the simulation is also revealed to be quite sensitive to the choice of $\mathrm{C} / \mathrm{H}$ ratio for the grey gas properties and adopting the higher value, corresponding to equal molar concentrations of the stable products $\mathrm{CO}_{2}$ and $\mathrm{H}_{2} \mathrm{O}$, reduces the incident flux too. The $\mathrm{C} / \mathrm{H}$ molar ratio of the MMA monomer is 0.625 and therefore lies outside the well-established values of 0.25 (methane) and 0.5 (fuel oil ) evaluated here [14]. The laminar flamelet calculation tends to displace the higher temperatures and product concentrations in the flame zone closer to the solid PMMA surface and this in turn marginally increases the incident radiative flux. The introduction of the flamelet model has greater impact on the computation of heat transfer, however, in relation to soot formation as described below. These simulations demonstrate that it is possible to bring about good agreement between prediction and experiment through the implementation of a number of different, but generally plausible, representations of the component models.

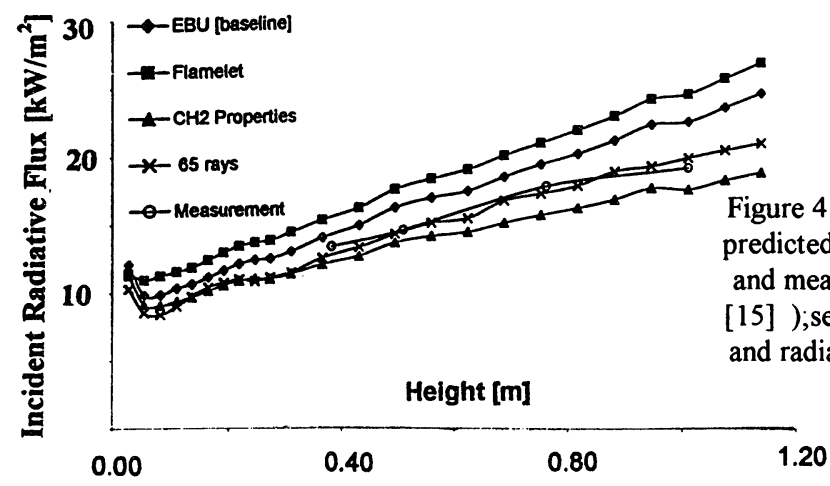

Additional detail - in the form of accompanying distributions of gas phase properties, for example - is necessary if these models are to be further discriminated. A rather similar observation can be made in relation to the influence of soot yield. All the preceding calculations simply prescribed the soot yield in the form of a fixed $(2 \%)$ conversion of the pyrolysed fuel flux to solid carbon at source. Figure 5 compares the resulting distribution of soot volume fraction, $f_{V}$, with that generated by the solution of additional balance equations for $f_{V}$ and $n$ as described earlier. In the flamelet-based calculation illustrated a single set of 
state relationships for the soot source terms,corresponding to a heat loss of $35 \%$, have been employed. Oxidation by $\mathrm{OH}$ [12] reduces the spread of the distribution in both the downstream direction, beyond the PMMA specimen, and transverse to the slab in comparison with that resulting simply from turbulent transport of soot as essentially an inert tracer. The predicted radiative flux incident on the slab is sensitive to the soot distribution as shown in fig. 6 , where the comparisons include a case in which oxidation has been entirely neglected and the soot concentration adjacent to the wall is approximately doubled. More detailed soot model evaluation however requires spatially resolved concentration measurements not available for these flame spread experiments.
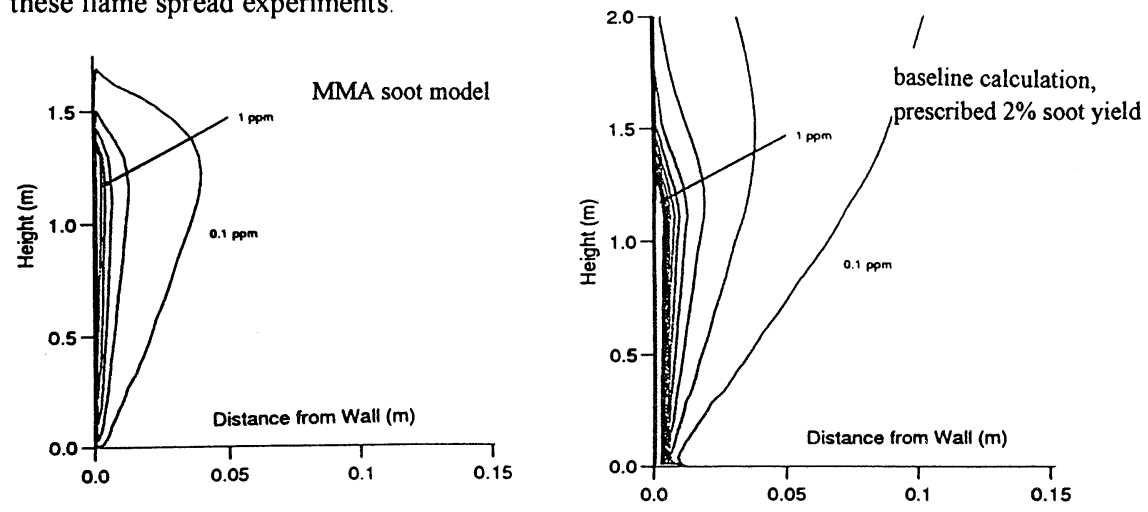

Figure 5 : Illustrative distributions of predicted soot volume fraction perpendicular to the pyrolysing slab

The pyrolysis model described is based on a comparatively simple extension of the unsteady heat conduction equation in the solid ( eqn.(5) ) but this demands a finely resolved computational mesh if the rate of surface mass release is to be satisfactorily reproduced. In the simulation of general three-dimensional fire scenarios this level of resolution may appear prohibitively expensive in relation to the computing resource available and alternatives are then necessary. The broad strategy, coupling a prescribed pyrolysis temperature to the detailed representations of the non-linear gas phase processes of combustion and heat transfer, does satisfactorily reproduce the physical flame spread behaviour observed experimentally however.

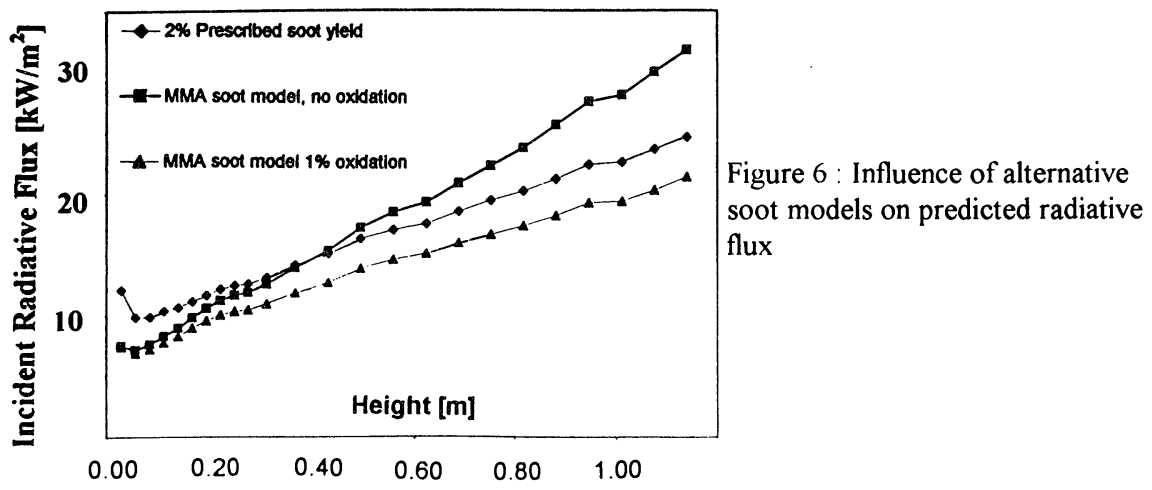


The introduction of analytical solutions for unsteady heat conduction of the kind illustrated in eqn.(1) to replace the solid phase computation has therefore been investigated. The simplified description of the thermal response of the solid which is envisaged must identify two key parameters - the time to surface ignition locally and the subsequent mass release rate. From the energy balance in eqn.(5) it is evident that the latter is particularly sensitive to the level of time-dependent internal heat conduction. Whilst the surface temperature during the inert heating phase can be approximated analytically from eqn.(1) such that, for example, under linearly increasing incident flux, $\dot{Q}$, the time to ignition, $t_{\imath g}$, becomes

$$
t_{i g}=\left(\frac{\lambda^{2} \pi}{\kappa}\right)\left(\frac{T_{p}-T_{0}}{4 / 3 \dot{Q}_{i g}}\right)^{2}
$$

the dynamics of the solid then play a larger part in determining the heat available for pyrolysis. For a semi-infinite solid with a fixed surface temperature $\left(T_{p}\right)$ the internal conduction may be described from [2] by

$$
\left.\frac{\partial T}{\partial x}\right|_{x=0}=\frac{T_{p}-T_{0}}{\sqrt{\pi \kappa t}}
$$

but whether the decay is better represented by the time, $t$, from ignition or the total elapsed time will depend on the slab heating history. In the two-dimensional flame spread experiment described earlier, where incident heat fluxes are comparatively low and the influence of slab heating is large, simple prescriptions based on such relationships are unable to reproduce the level of agreement with experiment already demonstrated in respect of transient flame spread and steady-state mass loss. At higher incident heat fluxes, more representative of room fires, this approach remains attractive, however, from both a computational standpoint and as a means of addressing complex surface materials. More elaborate solutions for the solid, introduced in parametric form, for example, are then necessary. These could perhaps be precomputed and stored in look-up tables, although the alternative, more computationallycentred, meshing options - based on multi-block or adaptive grids - appear more straightforward.

The base-line model described earlier has also been applied to the half-scale room corner fire configuration, incorporating PMMA walls $1 \mathrm{~m}$ high $\times 0.3 \mathrm{~m}$ wide $\times 0.02 \mathrm{~m}$ thick flush mounted within Marinite supporting walls and ceiling, investigated experimentally by Qian et al [17]. The corner walls were ignited at the base by a gas-fired torch, thereby minimising the preheating of the virgin material. Comparisons between prediction and experiment will here focus on two key parameters - the rate of upward spread of the pyrolysis front and the timedependent wall heat flux. The computational mesh is comparatively coarse, relative to the twodimensional simulation described earlier, but is broadly representative of that which might be achieved in a full compartment fire simulation - normal to the two PMMA surfaces there are initially 5 cells $/ \mathrm{cm}$, coarsening with distance from the wall to a $5 \mathrm{~cm}$ resolution; a similar distribution is employed vertically, progressively coarsening to a $10 \mathrm{~cm}$ resolution. The predicted spread of the pyrolysis front illustrated in fig.7, whilst capturing the essential features of the experiment, also reflects the limited resolution of the underlying grid. The rate of spread is generally underpredicted by approximately $20 \%$, as are the incident heat fluxes which are compared with measurement in fig. 8 . 

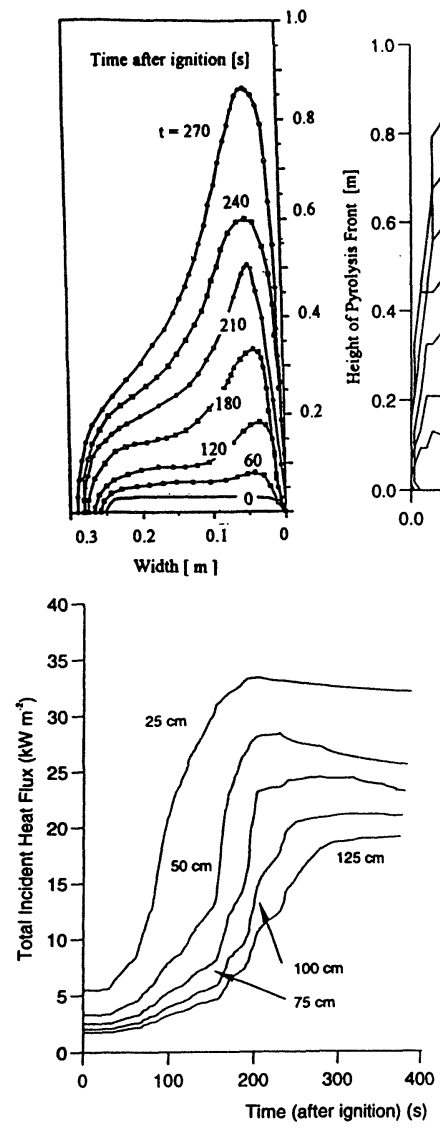

Figure 7 : Comparison between the predicted development of the pyrolysis front in the PMMA corner fire and the measurements of Qian et al [17]

Figure 8 : Comparison between the predicted distribution of incident heat flux and the measurements of Qian et al [17].

Whilst the model refinements described earlier might also improve these predictions, they are significantly more demanding in terms of spatial resolution. With room fire scenarios in mind, even the demonstrated level of agreement would appear sufficient to encourage the field modelling of fire growth, fuelled by surface flame spread, under more practically relevant conditions

\section{CONCLUSIONS}

A simplified model of non-charring solid pyrolysis has been coupled with detailed descriptions of turbulent combustion, soot production and radiative exchange to create a field model for fire spread prediction. The sensitivity of the predictions to alternative models and to the level of resolution employed is demonstrated by comparison with established two-dimensional experiments on PMMA slabs reported in the literature. The level of agreement between measurements of flame spread velocity, mass loss rate and incident heat flux is generally good. 
A baseline model incorporating the same components has also been applied to the more complex configuration of a room corner fire and again compared with experimental measurements in the literature. The level of resolution selected is one appropriate to more elaborate room fire scenarios and the agreement with experiment encourages the further extension to problems of more immediate practical interest

\section{ACKNOWLEDGEMENT}

The authors are pleased to acknowledge the financial support of EPSRC in the work reported and the many helpful discussions with members of the SOFIE Consortium.

\section{REFERENCES}

1. Fernandez-Pello, AC, The Solid Phase in Combustion Fundamentals of Fire ( ed. Cox,G ), Academic Press, 1995.

2. Carslaw, HS and Jaeger, JC, Conduction of Heat in Solids, Oxford University Press, 1959.

3. Babrauskas, V, Development of the Cone Calorimeter, Fire and Materials, $8,81-95,1984$.

4. Cox,G and Kumar, S, Field Modelling of Fire in Forced Ventilation Enclosures, Combust. Sci.Tech.

7, 7-23, 1987.

5. Kerrison,L, Galea,ER, Hoffmann, N and Patel,MK (1994) A Comparison of a FLOW3D Based Fire Field Model with Experimental Room fire Data, Fire Safety Jl, 23, 387-411, 1994

6. Lewis, MJ, Moss,JB and Rubini, PA, CFD Modelling of Combustion and Heat Transfer in Compartment Fires, Proc. Fifth Int Symp on Fire Safety Science,p.463-474, IAFSS, 1997.

7. Opstad, K, Modelling of Thermal Flame Spread on Solid Surfaces in Large Scale Fires, First European Symposium on Fire Safety Science, ETH Zurich, 1995.

8. Yan, Z, and Holmstedt,G , CFD Simulation of Upward Flame Spread over Fuel Surface, Proc. Fifth Int Symp on Fire Safety Science, p.345-356, IAFSS, 1997.

9. Spalding, DB, GENMIX : A General Computer Program for Two-Dimensional Parabolic Phenomena, Pergamon Press, 1977.

10. Bressloff, NW, Moss, JB and Rubini, PA, CFD Prediction of Coupled Radiation Heat Transfer and Soot Production in Turbulent Flames, Proc.Twenty-Sixth Int Symp on Combustion, p. 2379-2386, The Combustion Institute, 1996.

11. Moss, JB and Stewart, CD, Flamelet-based Smoke Properties for the Field Modelling of Fires, Fire Safety Jl, 30, 229-250, 1998.

12. Brookes, SJ and Moss, JB, Predictions of Soot and Thermal radiation Properties in Confined Turbulent Jet Diffusion Flames, Combust. Flame ,116, 486-503,1999.

13. Lockwood, FC and Shah, NG, A New Radiation Solution Method for Incorporation in General Combustion Prediction Procedures, Proc Eighteenth Int Symp on Combustion, p.1403-1413, The Combustion Institute, 1981.

14. Truelove, JS, Zone Method for Radiative Heat Transfer Calculations, HTFS DR33. AERE, Harwell, Oxon. England, 1975.

15. Orloff, L, De Ris, J, and Markstein, GH, Upward Turbulent Fire Spread and Burning of Fuel Surface, Proc. Fifteenth Int Symp on Combustion, p. 183-192, Combustion Institute, 1974.

16. Quintiere,JG and Rhodes,BT, Fire Growth Models for Materials, NIST-GCR-94-647. National Institute of Standards and Technology, 1994.

17. Qian, C, Ishida,H and Saito,K, Upward Flame Spread Along the Vertical Corner Walls, NISTGCR-94-648, National Institute of Standards and Technology, 1994.

18. Lewis, MJ Field Modelling of Flame Spread for Compartment Fire Prediction, PhD Thesis, Cranfield University, 1998.

19. Yan, $Z$ and Holmstedt,G, Fast Narrow Band Computer Model for Radiation Calculations, Numerical Heat Transfer Part B. 31, 61-71, 1997. 